\title{
NADPH oxidase-derived production of reactive oxygen species is involved in learning and memory impairments in 16-month-old female rats
}

\author{
HONGWEI KAN ${ }^{1,2^{*}}$, WEN HU ${ }^{1 *}$, YUCHAN WANG ${ }^{1}$, WANGYANG WU ${ }^{1}$, YANYAN YIN ${ }^{1}$, \\ YAN LIANG ${ }^{2}$, CHUNYAN WANG ${ }^{2}$, DAKE HUANG ${ }^{3}$ and WEIZU LI ${ }^{1}$
}

\begin{abstract}
${ }^{1}$ Department of Pharmacology, Key Laboratory of Anti-Inflammatory and Immunopharmacology, Ministry of Education, Anhui Medical University, Hefei, Anhui 230032; ${ }^{2}$ Laboratory of Pharmacology, Anhui Institute of Materia Medica, Hefei, Anhui 230022; ${ }^{3}$ Synthetic Laboratory of Basic Medicine College, Anhui Medical University, Hefei, Anhui 230032, P.R. China
\end{abstract}

Received May 14, 2014; Accepted April 4, 2015

DOI: $10.3892 / \mathrm{mmr} .2015 .3894$

\begin{abstract}
Women undergoing the natural menopause can experience progressive cognitive dysfunction, particularly in the form of memory impairment. However, the mechanisms underlying memory impairments in the menopause remain to be elucidated. There is increasing evidence that oxidative damage caused by excessive reactive oxygen species (ROS) production may correlate with age-associated cognitive impairment. The nicotinamide adenosine dinucleotide phosphate oxidase (NOX) family is important in the generation of ROS in the brain. It has been hypothesized that the accumulation of ROS, derived from NOX, may be involved in menopause-associated learning and memory impairments. The present study investigated whether NOX-derived ROS generation affected the learning and memory ability in 3-month and 16-month-old female rats. The results of a morris water maze assessment revealed that there were significant learning and memory impairments in the 16-month-old female rats. Furthermore, the activity of superoxide dismutase (SOD), level of malondialdehyde (MDA), production of ROS and expression levels of NOX2, p47phox, Ras-related C3 botulinum toxin substrate 1 ( $\mathrm{RAC1}$ ) and protein kinase $\mathrm{C} \alpha(\mathrm{PKC} \alpha)$ were investigated in the cortex and hippocampus of 3-month and 16-month old female rats. The results demonstrated that the activity of SOD was significantly decreased, whereas the levels of MDA, production of ROS and expression levels of NOX2, p47phox,
\end{abstract}

Correspondence to: Dr Weizu Li, Department of Pharmacology, Key Laboratory of Anti-Inflammatory and Immunopharmacology, Ministry of Education, Anhui Medical University, 81 Meishanlu, Hefei, Anhui 230032, P.R. China

E-mail: liweizu@126.com

${ }^{*}$ Contributed equally

Key words: menopause, learning and memory impairments, reactive oxygen species, NADPH oxidase, Morris water maze
$\mathrm{RAC} 1$ and PKC $\alpha$ were significantly increased in the 16-month old female rats. These results suggested that NOX-mediated oxidative stress may be important in menopause-associated learning and memory impairments.

\section{Introduction}

Natural menopause is a gradual process, which occurs for the majority of women between the ages of 47 and 55 years (1). During the menopause transition, the majority of women report memory problems (2). Numerous studies have revealed that females undergoing surgical or natural menopause may experience memory impairment $(3,4)$, which has been reproduced in adult ovariectomized female rats and mice $(5,6)$. Previous studies have indicated that unilateral and bilateral oophorectomy preceding the onset of the menopause is associated with an increased risk of cognitive impairment or dementia $(3,4)$. This risk was observed to increase with younger age at oophorectomy (7). However, the mechanisms underlying memory impairment in the menopause remain to be elucidated. A previous study demonstrated that a low level of endogenous estrogen in the serum was associated with increased risk of poor verbal memory (8), while other studies have failed to confirm this $(9,10)$. These reports suggest that there are other mechanisms, which are involved in cognitive impairments in menopause.

There is increasing evidence that oxidative damage caused by excess reactive oxygen species (ROS) may correlate with several neurodegenerative diseases, including Alzheimer's disease and Parkinson's disease (11-13). Excessive ROS can cause oxidative damage via the modification of proteins, lipids and DNA, and the brain is particularly vulnerable to the oxidative damage caused by ROS as it consumes large quantities of oxygen and has an abundant lipid content, but a relative paucity of antioxidant compounds, compared with other organs $(14,15)$.

There are multiple potential sources for ROS in mammalian cells, including the mitochondrial electron transport chain, xanthine oxidase, cyclooxygenases and monoamine oxidases $(16,17)$. However, the nicotinamide adenosine 
dinucleotide phosphate oxidase enzyme (NADPH oxidase; NOX) is important, as it is dedicated to the specific production of superoxide. Superoxide production via NADPH oxidase has been demonstrated to be important in a variety of neurological disorders, including Alzheimer's disease. NOX is composed of membrane-bound (gp91phox, also termed NOX2 and $\mathrm{p} 22 p h o x)$ and cytoplasmic subunits [p40phox, p47phox, p67phox and Ras-related C3 botulinum toxin substrate 1 (RAC1)] (18). NOX2 is expressed throughout the central nervous system and its levels are particularly high in neurons, astrocytes and microglia (19). NOX2 has been implicated in nerve growth factor signaling and may be important in the plasticity of the nervous system, including learning and memory formation (20). Previous studies have demonstrated that the p67phox, p47phox and p40phox NOX cytosolic subunit proteins are significantly elevated with the progression of Alzheimer's disease $(18,20)$. In addition, a marked correlation has been observed between NOX activity and cognitive status, in which as NOX activity increased, cognitive performance decreased (18). The present study hypothesized that oxidative damage caused by NADPH oxidase-derived ROS accumulation may be involved in the learning and memory impairments in menopause, and learning and memory abilities, the activity of superoxide dismutase (SOD), the level of malondialdehyde (MDA) and NADPH oxidase-derived ROS accumulation were investigated in 3-and 16-month-old female rats to confirm this hypothesis.

\section{Materials and methods}

Animals and treatment. The animal experiments were approved by the Institutional Animal Care and Use Committee of Anhui Medical University (Hefei, China). Sprague Dawley rats were obtained from the Shanghai B\&K Universal Group Limited (Shanghai, China). Female adolescent rats (3 months; 210-260 g) and female aged rats (16-months; 490-540 g) rats were used in the present study (17 animals in each group). All animals were housed in standard cages with a $12 \mathrm{~h}$ light/dark cycle and had ad libitum access to food and water throughout the investigation. The animals were allowed to acclimate to their new surroundings for 1 week prior to the commencement of the behavioral experiments.

Morris water maze. The water maze consisted of a plastic black circular tank ( $160 \mathrm{~cm}$ in diameter; $60 \mathrm{~cm}$ high). An overhead video camera connected to a computer and ANY-maze video tracking system software (Stoelting Company, Wood Dale, IL, USA) was used to track the path of movement of the rat. The water was maintained at a constant temperature of $22-25^{\circ} \mathrm{C}$. A circular escape platform $(10 \mathrm{~cm}$ diameter) was placed $2 \mathrm{~cm}$ below the surface of the water, in the middle of one of the four quadrants. The general assessment process has been described in detail previously $(21,22)$. In the training trials, the animals were placed into the tank from the four quadrants each day, with a 5 min trial interval, for 4 days. Each trial lasted until the rats climbed onto the hidden platform within $90 \mathrm{sec}$, the rats were guided to the platform if they had not climbed onto the platform within $90 \mathrm{sec}$. The escape latency onto the platform was recorded to indicate the learning performance. Following the training trials, the platform was removed from the pool and each rat received one swim probe trial of $60 \mathrm{sec}$. The swimming distance (m) and the mean speed $(\mathrm{m} / \mathrm{sec})$ were recorded as an indication of motor behavior. The swimming duration in the quadrant of the platform (sec), swimming distance in the quadrant of the platform (m), number of crossings of the platform and time of first entry onto the platform (sec) were recorded as an indicator of memory aptitude.

Levels of serum estradiol and luteinizing hormone ( $\mathrm{LH})$. The levels of serum estradiol and LH were measured using an automated microparticle enzyme immunoassay kit and analysis instrument (AxSYM; Abbott Diagnostics, Inc., Abbott Park, IL, USA). The AxSYM method is a heterogeneous immunoassay, in which estradiol and LH from the specimens bind to rabbit polyclonal anti-estradiol antibodies and anti-LH antibodies, which are linked to microparticles. Following removal of the unbound materials, estradiol and LH alkaline phosphatase conjugate were added and bound to available sites. Following washing with PBS, 4-methylumbelliferyl phosphate was added and the fluorescent product was measured. In this method, the intensity of the signal is inversely proportional to the concentrations of estradiol and LH in the specimens $(23,24)$.

Activity of SOD and content of MDA in brain tissue. The animals were sacrificed via cervical dislocation (eight rats in each group). The brain was immediately dissected in half along the coronal line, one half of which was frozen $\left(-8^{\circ} \mathrm{C}\right)$ for immunoblot analysis and the other was used to form a $10 \%$ physiological saline homogenate. The homogenate was centrifuged at $4,000 \mathrm{xg}$ for $10 \mathrm{~min}$, and the supernatant was collected. The activity of SOD and the level of MDA were detected using a spectrophotometer (SpectraMax 190; Molecular Devices Corp., Sunnyvale, CA, USA), according to the SOD and MDA assay kits (Niajing Jiancheng Bioengineering Institute, Nanjing, China).

Production of ROS in the frontal cortex and hippocampus. Dihydroethidium (DHE) microfluorography (Beyotime Institute of Biotechnology, Haimen, China) was used to determine the production of ROS in the brain tissue (25). DHE is a cell permeable dye, which is oxidized to ethidium bromide and associated products by superoxide. Ethidium bromide is trapped intracellularly by intercalation into DNA and can then be identified using red fluorescence $(26,27)$. In the present study, DHE was injected via the vena caudalis $(100 \mu \mathrm{M} ; 1 \mathrm{ml} / 100 \mathrm{~g})$ for $30 \mathrm{~min}$ prior to sacrifice of the rat $(n=8$; four rats/group). The brains were removed from the skull and transferred to a freezer at $-20^{\circ} \mathrm{C}$. Subsequently, the brains were sectioned at a thickness of $20 \mu \mathrm{m}$ using a cryostat section cutter (Leica, Wetzlar, Germany) at $-20^{\circ} \mathrm{C}$ and mounted onto glass slides. The sections were washed with phosphate buffered saline three times and examined using a fluorescence microscope (Olympus IX71; Olympus, Tokyo, Japan) equipped with a custom filter set for detection of DHE oxidation products (red). The production of ROS in the cortex and the hippocampal CA1 and CA 3 regions were assessed using previously described methods (25). The exposure time was $900 \mathrm{~ms}$ for all sections. A total of four sections 
per group were assessed for ROS quantitative analysis. The mean fluorescence intensities of three consecutive fields (magnification, x400) in the cortex, and hippocampal CA1 and $\mathrm{CA} 3$ regions, were performed in a blinded-manner using the Image-Pro Plus 6.0 (Media Cybernetics, Inc., Silver Spring, MD, USA) analysis system to indicate the production of ROS, with the data expressed in relative fluorescence units.

Immunohistochemistry. Following the morris water maze assessment, the animals ( $\mathrm{n}=10$; five rats/group) were sacrificed and the brains were removed. The brains were fixed in $4 \%$ paraformaldehyde and embedded in paraffin. The paraffin sections were cut at $5 \mu \mathrm{m}$ and mounted onto glass slides. Prior to immunostaining, the sections were deparaffinized and rehydrated in water. The endogenous peroxidase activity was inhibited by incubation with $0.3 \%$ hydrogen peroxide for $30 \mathrm{~min}$. Subsequently, the sections were incubated with normal goat serum at room temperature for $30 \mathrm{~min}$, following which the sections were incubated with primary antibodies overnight at $4^{\circ} \mathrm{C}$. The primary rabbit polyclonal antibodies were protein kinase C $\alpha$ (PKC $\alpha$; BS1577; 1:200; Bioworld Technology, St. Louis Park, MN, USA), p47phox (BS4600; 1:200; Bioworld Technology), NOX2 (BS5674; 1:100; Bioworld Technology) and RAC1 (24072-1-AP; 1:100; ProteinTech, Chicago, IL, USA). Immunostaining was visualized using a peroxidase method with an ABC kit (Beijing Zhongshan Golden Bridge Biotechnology Co., Ltd., Beijing, China). The sections were resin-mounted and observed under a microscope (Olympus IX71; Olympus). The positive cells were stained brown. A total of five sections in each group were assessed for quantitative analysis and a total of three consecutive fields (magnification, $\mathrm{x} 400$ ) of the cortex, and hippocampal CA1 and CA3 regions, in each section were observed. The mean optical density of the positive neurons of the hippocampal CA 1 and CA 3 regions and the cortex in each section were measured using the Image-Pro Plus 6.0 analysis system to determine the expression levels of PKC $\alpha$, p47phox, NOX2 and RAC1 (22).

Immunoblot analysis. Tissue from the cortex and the hippocampus (100 mg) were homogenized in $1 \mathrm{ml}$ radioimmunoprecipitation assay lysis buffer; Beyotime Institute of Biotechnology). Equal quantities of protein $(50 \mu \mathrm{g})$ were separated using SDS-PAGE and transferred onto polyvinylidene difluoride membranes. The membranes were washed in Tris-buffered saline with Tween 20 (TBS-T) and further incubated with the antibodies against NOX2 (1:500), p47phox (1:500), RAC1 $(1: 500)$ and $\beta$-actin $(1: 1,000)$ overnight at $4^{\circ} \mathrm{C}$. The membranes were thoroughly washed with TBS-T and then incubated with anti-rabbit immunoglobulin $\mathrm{G}$ antibody conjugated to horseradish peroxidase $(1: 10,000)$ for $1 \mathrm{~h}$. Following washing with TBS-T, the immunoreactive bands were visualized using an enhanced chemiluminesense kit (Amersham Biosciences, Little Chalfont, UK). The Tanon4500 imaging system (Shanghai Tanon Science \& Technology Co., Ltd., Shanghai, China) was used to visualize protein bands, and densitometry was performed using Image J software, version 1.43 (National Institutes of Health, Bethesda, MD, USA). The ratio of the target band to that of $\beta$-actin was determined to calculate the relative intensity of the expression of p47phox, NOX2 and RAC1.
Statistical analysis. Data are expressed as the mean \pm standard deviation. The SPSS 17.0 (SPSS, Inc., Chicago, IL, USA) statistical software package was used to analyze the results. Statistical comparisons between groups were performed using Student's t-test with Bonferroni's post hoc test. $\mathrm{P}<0.05$ was considered to indicate a statistically significant difference.

\section{Results}

Learning and memory impairments in 16-month-old female rats, determined using a Morris water maze. A Morris water maze assessment was performed to assess the learning and memory skills in the rats, as described above. In the learning and memory training experiments, the mean escape latencies in the 3 -and 16-month-old groups on the first day were significantly different (40.02 \pm 13.74 , vs. 58.89 \pm 8.75 , respectively; $\mathrm{P}=0.005)$. No significant differences were observed in the training experiments between day 2 and day 4 in the 3-month and 16-month-old groups (Fig. 1A). In the probe trial experiments (Fig. 1B), the swimming distance $(14.16 \pm 1.56$, vs. $11.30 \pm 1.90 ; \mathrm{P}=0.007$; Fig. 1C), mean swimming speed $(0.24 \pm 0.03$, vs. $0.19 \pm 0.03$; $\mathrm{P}=0.007$; Fig. 1D), and the swimming duration and distance to the platform (Fig. 1E and $1 \mathrm{~F} ; \mathrm{P}=0.006$ and $\mathrm{P}=0.003$ ) were significantly different in the 3-month and 16-month-old groups, respectively. In addition, the number of crossings $(3.88 \pm 1.55$, vs. $2.13 \pm 1.3$; $\mathrm{P}=0.035$; Fig. $1 \mathrm{G})$ and the time of first entry onto the platform $(13.71 \pm 12.76$, vs. $35.01 \pm 17.74 ; \mathrm{P}=0.02$; Fig. $1 \mathrm{H})$ in the 3-month and 16-month-old groups, respectively were significantly different.

Levels of serum estradiol and $\mathrm{LH}$ in the 16-month-old rats. Levels of estradiol, which decline during the menopause have been associated with learning and memory impairments $(28,29)$. To investigate the possible links between memory impairments in the menopause and the levels of serum estradiol and $\mathrm{LH}$, the levels of serum estradiol and $\mathrm{LH}$ in the 3-month and 16-month-old rats were examined. The results revealed that, compared with the 3-month-old rats, the level of serum estradiol was significantly decreased in the 16-month-old rats $(137.86 \pm 26.10$, vs. $91.15 \pm 25.99$, respectively; $\mathrm{P}=0.0008$; Fig. 2A). No significant differences were identified in the level of serum LH between the 3-month and 16-month-old rats (Fig. 2B).

Activity of SOD and the level of MDA in the brain tissues. Compared with the 3-month-old group, the SOD activity was significantly decreased $(\mathrm{P}=0.03)$ and the MDA content was significantly increased $(\mathrm{P}=0.009)$ in the 16 -month-old rats (Fig. 3).

ROS production in the frontal cortex and hippocampal CA1 and CA3 regions in 16-month old female rats. DHE was used to detect the production of ROS in the cortex and hippocampal CA1 and CA3 regions in the 3-month and 16-month-old rats (27). A low level of fluorescence was detected in the cortex and hippocampal CA1 and CA3 regions in the 3-month rats (Fig. 4A), indicating mild ROS production. However, in the 16-month-old group, the production of ROS was significantly increased in the cortex and hippocampal $\mathrm{CA} 1$ and $\mathrm{CA} 3$ regions (Fig. 4A and 4B; $\mathrm{P}=0.001, \mathrm{P}=0.024$ and $\mathrm{P}=0.009$, respectively). 


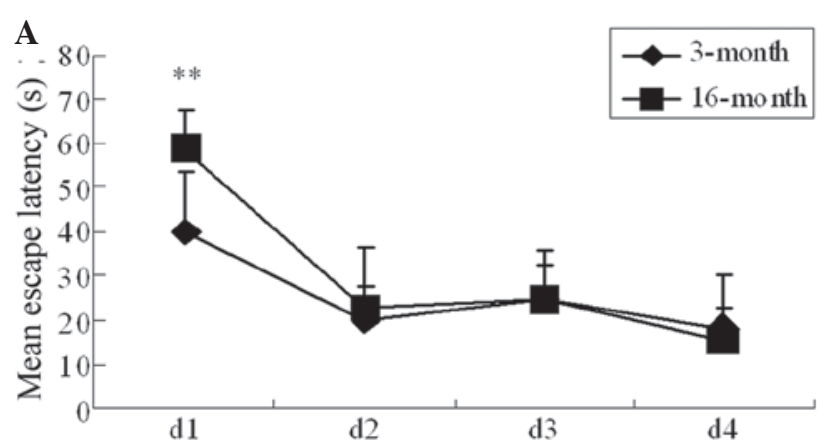

C

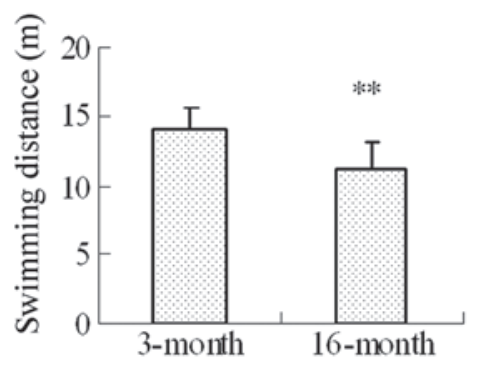

F

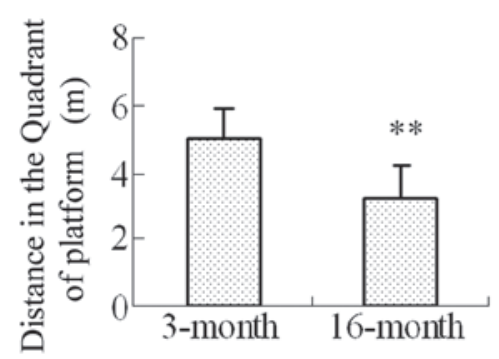

D

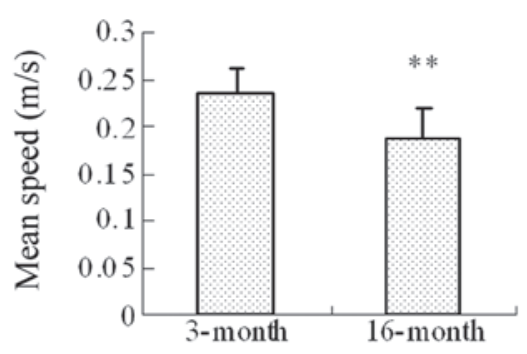

G

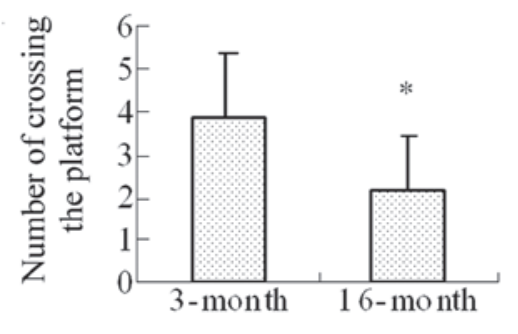

B

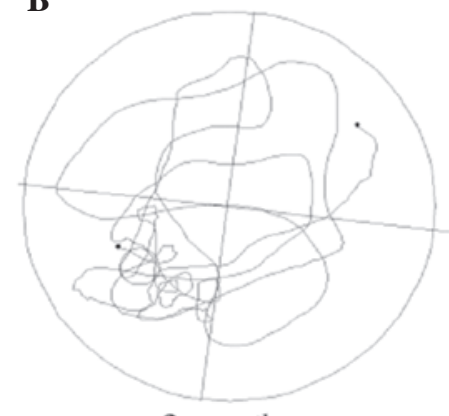

3-month

$\mathbf{E}$

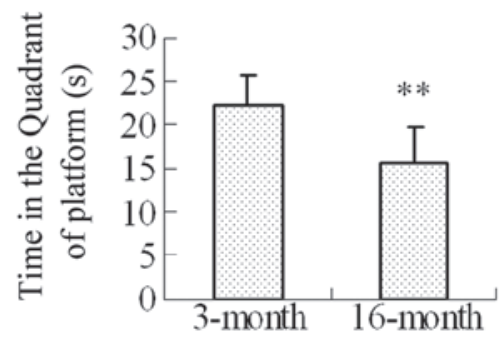

$\mathbf{H}$

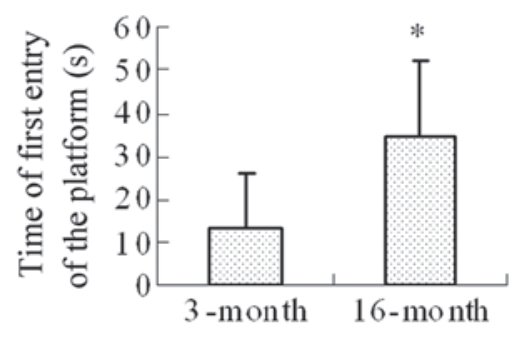

Figure 1. Learning and memory abilities of the 3-month and 16-month-old female rats in the Morris water maze task. (A) Learning and memory training experiment over 4 days. (B) Representative path of probe trial experiments on day 5. (C) Swimming distance. (D) Mean swimming speed. (E) Swimming duration in the quadrant of the platform. (F) Distance of the platform in the quadrant. (G) Number of crossings of the platform. (H) Time of first entry onto the platform. Data are expressed as the mean \pm standard deviation, $\mathrm{n}=8,{ }^{*} \mathrm{P}<0.05$ and ${ }^{* * *} \mathrm{P}<0.01$, vs. 3 -month old group.

A

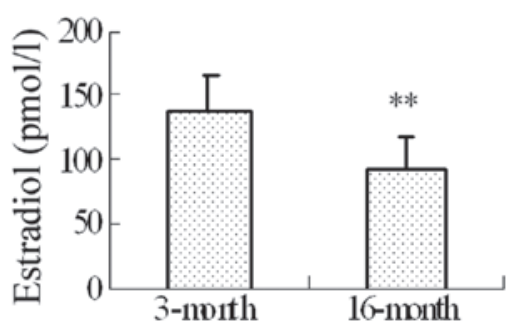

B

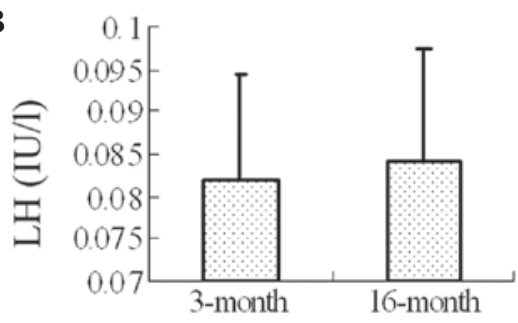

Figure 2. Levels of plasma estradiol and LH in 3-month and 16-month-old female rats. (A) Level of plasma estradiol. (B) Level of plasma LH. Data are expressed as the mean \pm standard deviation, $\mathrm{n}=8,{ }^{* *} \mathrm{P}<0.01$, vs 3 -month group. $\mathrm{LH}$, luteinizing hormone.

A

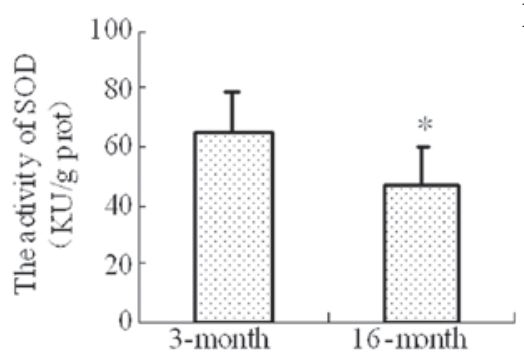

B

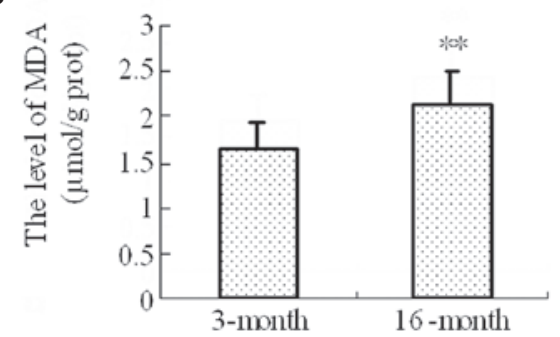

Figure 3. Activity of SOD and the level of MDA in the brain tissue of the 3-month and 16-month-old female rats. (A) Activity of SOD. (B) Level of MDA. Data are expressed as the mean \pm standard deviation, $n=8,{ }^{*} \mathrm{P}<0.05$ and ${ }^{* *} \mathrm{P}<0.01$, vs. 3 -month group. MDA, malondialdehyde; SOD, superoxide dismutase. 
A

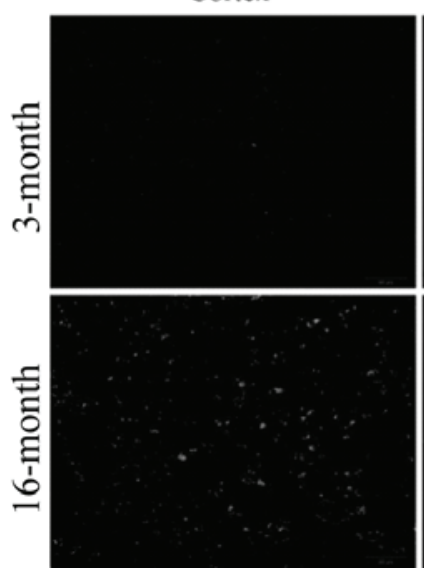

CAl

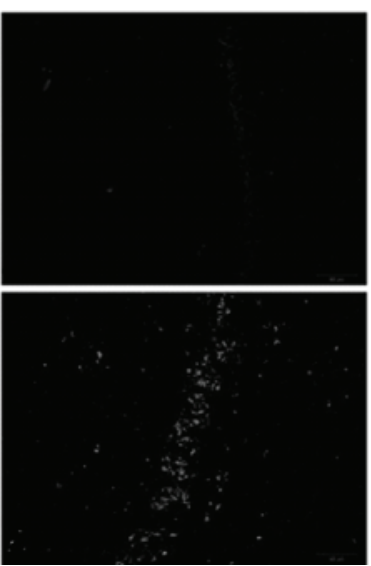

CA3

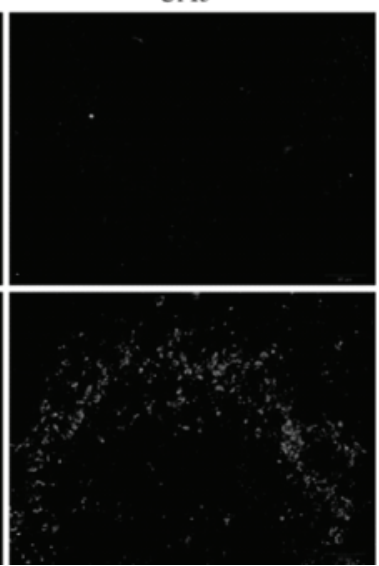

B

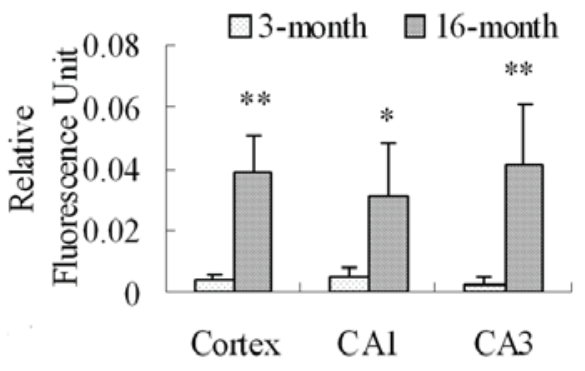

Figure 4. ROS production in the cortex and hippocampal CA1 and CA3 regions in 3-month and 16-month-old female rats (dihydroethidium fluorescence, magnification, $\mathrm{x} 400$ ). (A) ROS production in the cortex and - hippocampal CA1 and CA3 regions. (B) Quantitative analysis of the ROS production in the cortex and - hippocampal CA1 and CA3 regions. Data are expressed as the mean \pm standard deviation, $\mathrm{n}=4,{ }^{*} \mathrm{P}<0.05$ and ${ }^{* *} \mathrm{P}<0.01$, vs. 3 -month group. ROS, reactive oxygen species.

Expression levels of PKC $\alpha, N O X 2, p 47$ phox and RAC1 in the frontal cortex and hippocampus in 16-month-old rats. NOX family members are important sources for the generation of ROS in neurodegenerative diseases. Therefore, the expression levels of PKC $\alpha$, which activates NOX2, the NOX2 membrane subunit, the p47phox cytosolic subunit and RAC1 were investigated in the frontal cortex and hippocampal CA1 and CA3 regions using immunohistochemistry. The $\mathrm{PKC} \alpha$ immunostaining results revealed that the expression of PKC $\alpha$ was significantly increased in the cortex and the hippocampal CA1 and CA3 regions in the 16-month-old group (Fig. 5A and 5B; $\mathrm{P}<0.05$ ). The NOX2 immunostaining results revealed lower expression levels of NOX2 in the cortex and the hippocampal CA1 and CA3 regions in the 3-month-old rats, as the immunoreactive staining was light. Compared with the 3-month old group, the numbers of NOX2 immunoreactive cells were significantly increased in the cortex and the hippocampal CA1 and CA3 regions in the 16-month-old rats, in which staining was marked (Fig. 5C and 5D; $\mathrm{P}<0.05)$. The p47phox and $\mathrm{RAC} 1$ immunostaining results also revealed that the p47phox cytosolic subunit and RAC1, particularly p47phox, were expressed in the cortex and hippocampus in the two groups. However, compared with the 3-month group, the expression levels of $\mathrm{p} 47$ phox and RAC1, particularly p47phox, were significantly increased in the cortex and the hippocampal CA1 and CA3 regions in the 16-month old female rats (Fig. 5E and 5F; $\mathrm{P}<0.05$ and $\mathrm{P}<0.01$ for p47phox; Fig. $5 \mathrm{G}$ and $5 \mathrm{H}$; $\mathrm{P}<0.05$ for $\mathrm{RAC1}$ ).

The expression levels of NOX2, p47phox and RAC1 were also assessed in the frontal cortex and the hippocampus using immunoblotting (Fig. 6A). The results revealed that the expression levels of NOX2, p47phox and RAC1 were also significantly increased in the 16-month-old rats (Fig. 6B; $\mathrm{P}<0.05)$.

\section{Discussion}

In the present study, menopause-associated differences in learning and memory ability, levels of serum estradiol and
LH, and NADPH oxidase-derived ROS production were examined in the frontal cortex and hippocampal CA1 and CA3 regions of 3-month and 16-month-old female rats. The natural menopause is characterized by a progressive decline in the levels of estrogen and is often accompanied by locomotor decline with age $(8,28,30)$. Numerous studies have demonstrated that there are significant learning and memory impairments in females undergoing surgical or natural menopause and in adult ovariectomized female rats and mice $(4,5,31,32)$. In addition, estrogen levels can affect learning and memory, inducing impairments in spatial reference memory, spatial working memory and non-spatial memory, in aging and adult rodents (3,31-33). The present study revealed that learning and memory abilities were significantly decreased in the 16-month-old female rats. In addition, the level of serum estradiol was found to be significantly decreased in the 16-month-old rats. These results were consistent with those of previous studies (3,31-33). However, these changes are also associated with age and further studies are required to elucidate the association between menopause and age-associated learning and memory impairments.

In 1956, Harman (34) introduced the free radical theory of aging, suggesting that constitutively produced ROS interact with cellular components in a cumulatively deleterious manner. Subsequent studies have demonstrated that ROS are central in the pathogenesis of several diseases, including cardiovascular disease, kidney damage and neurodegenerative diseases (35-37). The cortex and the hippocampus are important brain areas, involved in the process of learning and memory. Whether ROS levels in the cortex and the hippocampus are involved in the modulation of learning and spatial memory decline in the menopause remains to be elucidated. In the present study, the activity of SOD, the level of MDA and the production of ROS were examined in the cortex and hippocampus of 3-month and 16-month-old rats. The results revealed that the activity of SOD was decreased and the content of MDA was increased in the brain tissues of the 16-month-old rats. ROS production was also increased significantly in the cortex and the hippocampal CA1 and CA3 regions in the 16-month-old female rats. These data 
A

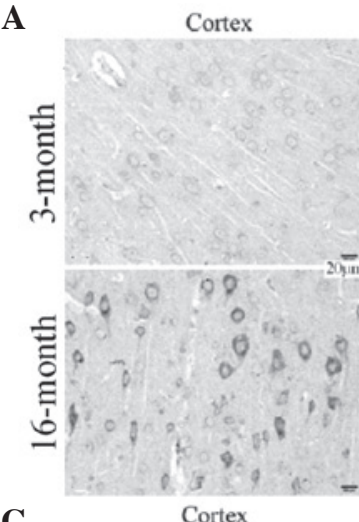

C

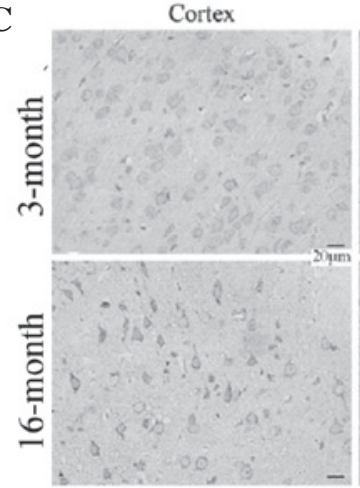

$\mathbf{E}$

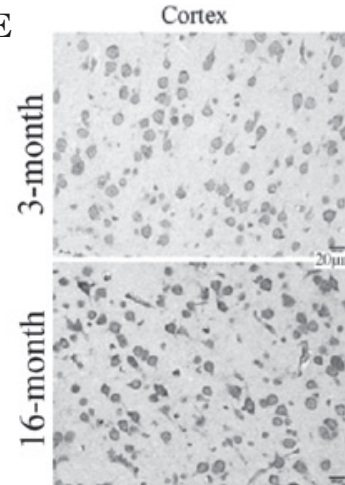

G

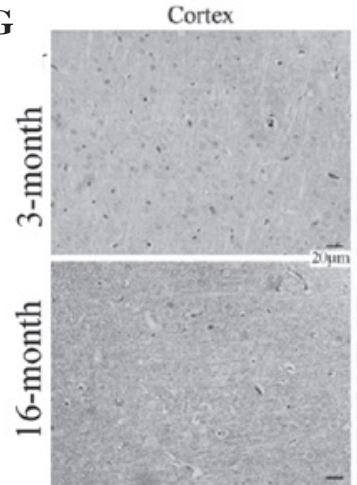

CAI

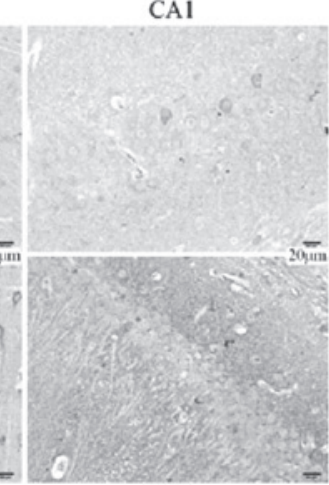

CAI

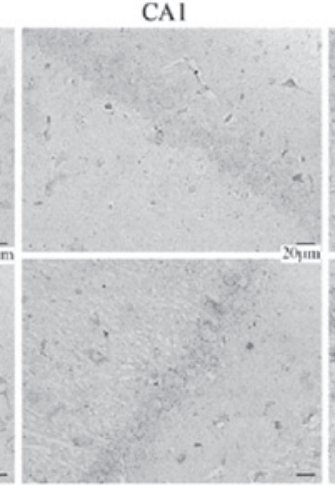

CAI

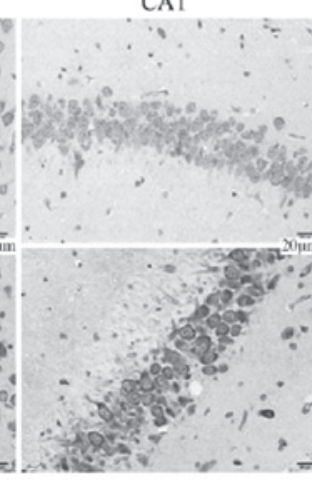

CA1

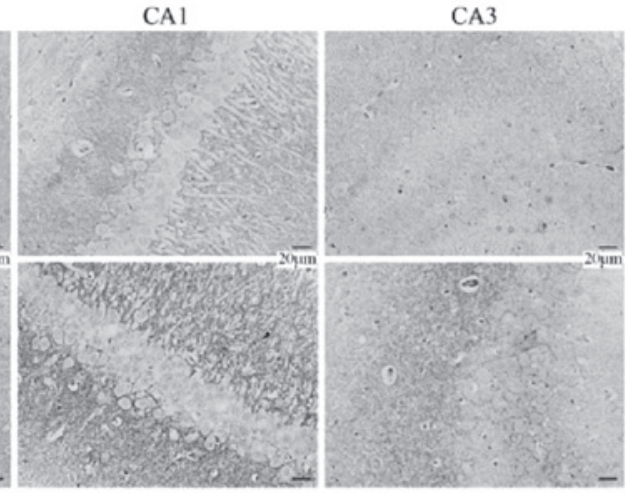

B

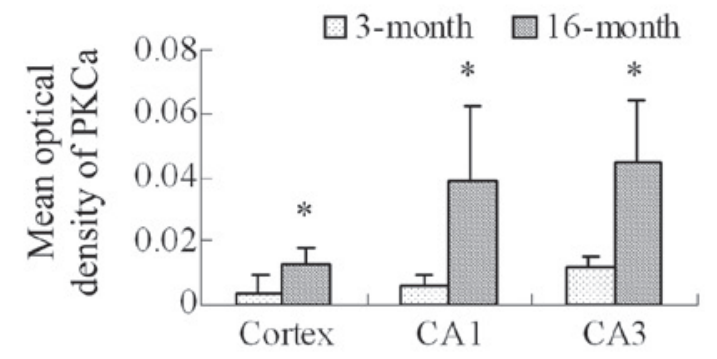

D

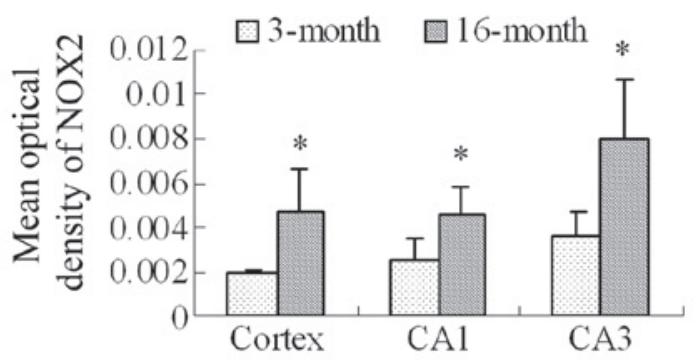

$\mathbf{F}$

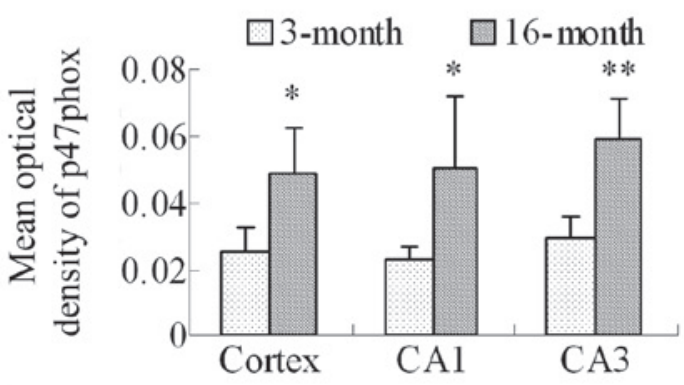

$\mathbf{H}$

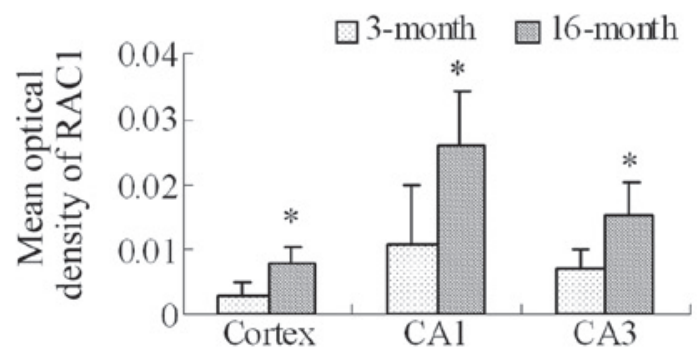

Figure 5. Expression levels of PKC $\alpha$, NOX2,p47phox and RAC1 in the frontal cortex and hippocampal CA1 and CA3 regions in the 3-month and 16-month-old female rats (immunostaining, magnification, $\mathrm{x} 400$ ). The darker areas indicate immunoreactive neurons. (A) Expression of PKC $\alpha$ in the frontal cortex and hippocampal CA1 and CA3 regions. (B) Quantitative analysis of the expression of PKC $\alpha$. (C) Expression of NOX2 in the frontal cortex and hippocampal CA1 and CA3 regions. (D) Quantitative analysis of the expression of NOX2. (E) Expression of p47phox in the frontal cortex and hippocampal CA1 and CA3 regions. (F) Quantitative analysis of the expression of p47phox. (G) Expression of RAC1 in the frontal cortex and hippocampal CA1 and CA3 regions. (H) Quantitative analysis of the expression of RAC1. Data are expressed as the mean \pm standard deviation, $\mathrm{n}=5,{ }^{*} \mathrm{P}<0.05$ and ${ }^{* *} \mathrm{P}<0.01$, vs. 3-month group. PKC $\alpha$; Protein kinase $\mathrm{C} \alpha$; NOX2, nicotinamide adenine dinucleotide phosphate-oxidase 2; RAC1, Ras-related C3 botulinum toxin substrate 1.

suggested that the accumulation of ROS in the cortex and the hippocampus was important in the modulation of learning and memory impairments in the menopause.
There is increasing evidence that NOX family members are important sources of ROS in neurodegenerative diseases $(38,39)$. Previous studies have reported a correlation 


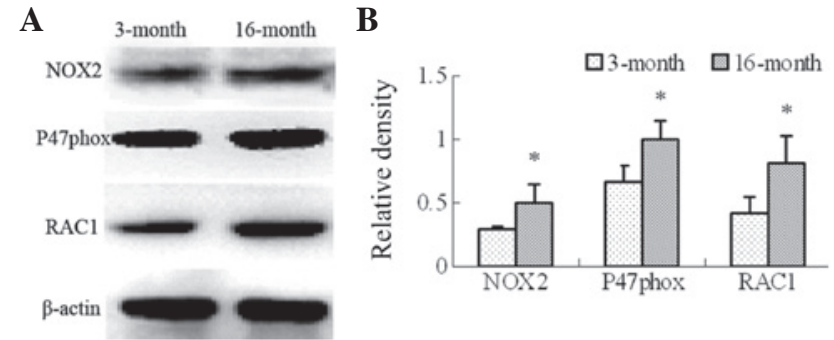

Figure 6. Corresponding densitometry for the expression levels of NOX2, p47phox and RAC1 relative to $\beta$-actin. (A) Immunoblot of the expression of NOX2, p47phox and RAC1 in the frontal cortex and hippocampus. (B) Corresponding densitometry for the expression levels of NOX2, p47phox and RAC1 relative to $\beta$-actin. Data are expressed as the mean \pm standard deviation, $\mathrm{n}=4,{ }^{*} \mathrm{P}<0.05$, vs.3-month group. RAC1, Ras-related $\mathrm{C} 3$ botulinum toxin substrate 1; NOX2, nicotinamide adenine dinucleotide phosphate-oxidase 2 .

between NOX activity and the progression of Alzheimer's disease, suggesting that increased NADPH oxidase activity may be one of the early events in the transition from normal cognition to dementia $(18,40,41)$. Furthermore, the increased expression of NOX4 in human renin/angiotensinogen chimeric transgenic mice is associated with increased NOX activity and cognitive decline (42). In addition, NOX activity is also significantly increased in aged amyloid precursor protein/presinilin 1 mice, and shares a significant linear correlation with deficits in cognitive function (17). However, whether NOX-mediated ROS accumulation is involved in the cognitive impairments in the menopause remains to be elucidated.

NOX, which is dedicated to the specific production of ROS, consists of membrane (gp91phox, also termed NOX2 and p22phox) and cytosolic (p47phox, p67phox, p40phox and RAC1) components $(43,44)$. The NOX2 membrane-integrated protein is the catalytic core of the enzyme responsible for electron transfer between NADPH and molecular oxygen for superoxide production. In nonphagocytic cells, NOX is constitutively activated, producing relatively low levels of ROS under basal conditions and generating higher levels of ROS in response to cytokines and growth factors. The increased ROS accumulation can result in the stimulation of redox-sensitive intracellular signaling pathways (45). Previous studies have revealed that PKC can phosphorylate the gp91phox-cytosolic tail, and phosphorylation of gp91phox enhances the catalytic activity and assembly of the complex (46). Upon activation, the cytosolic components translocate to the membrane, as a result of interactions between the cytosolic components and its phospholipid environment (47). A previous study demonstrated that the elevated expression of $\mathrm{p} 47$ phox was confirmed as NOX activation (48). In the present study, the immunohistochemistry results revealed that the expression levels of PKC $\alpha$, NOX2, p47phox and RAC1 were significantly increased in the cortex and the hippocampus of 16-month-old female rats, compared with 3-month-old rats. The immunoblotting results also demonstrated that the expression levels of NOX2, p47phox and RAC1 were significantly increased in the 16-month-old female rats. These data suggested that NOX-derived ROS accumulation in the frontal cortex and hippocampus may be involved in cognitive impairments in 16-month-old female rats.
In conclusion, the present study demonstrated that NOX-derived ROS accumulation may be involved in menopause-associated learning and memory impairments. Although the present study provided an experimental basis for the mechanism of NOX-derived ROS accumulation and learning and memory impairments in 16 month old female rats, further investigations are required to elucidate the mechanisms underlying menopause-associated learning and memory impairments.

\section{Acknowledgements}

This study was supported by the National Natural Science Foundation of China (grant no. 81371329), the Nature Science Foundation of Anhui Province (grant. no. 1308085MH144) and the Doctor Foundation of Anhui Medical University (grant no. XJ201011). The authors would like to thank Mr. Shan Huang and Mrs. Li Gui from the Synthetic Laboratory of Basic Medicine College, Anhui Medical University for their technical assistance.

\section{References}

1. Greendale GA, Lee NP and Arriola ER: The menopause. Lancet 353: 571-580, 1999.

2. Sullivan Mitchell E and Fugate Woods N: Midlife women's attributions about perceived memory changes: observations from the Seattle Midlife Women's Health Study. J Womens Health Gend Based Med 10: 351-362, 2001.

3. Sherwin BB: Surgical menopause, estrogen and cognitive function in women: what do the findings tell us? Ann NY Acad Sci 1052: 3-10, 2005.

4. Henderson VW and Popat RA: Effects of endogenous and exogenous estrogen exposures in midlife and late-life women on episodic memory and executive functions. Neuroscience 191: 129-138, 2011.

5. Luine VN, Richards ST, Wu VY and Beck KD: Estradiol enhances learning and memory in a spatial memory task and effects levels of monoaminergic neurotransmitters. Horm Behav 34: 149-162, 1998.

6. Frick KM, Fernandez SM and Bulinski SC: Estrogen replacement improves spatial reference memory and increases hippocampal synaptophysin in aged female mice. Neuroscience 115: 547-558, 2002.

7. Rocca WA, Bower JH, Maraganore DM, Ahlskog JE Grossardt BR, de Andrade $\mathrm{M}$ and Melton LJ: Increased risk of cognitive impairment or dementia in women who underwent oophorectomy before menopause. Neurology 69: 1074-1083, 2007.

8. Manly JJ, Merchant CA, Jacobs DM, Small SA, Bell K, Ferin M and Mayeux R: Endogenous estrogen levels and Alzheimer's disease among postmenopausal women. Neurology 54: 833-837, 2000.

9. Phillips SM and Sherwin BB: Effects of estrogen on memory function in surgically menopausal women. Psychoneuroendocrinology 17: 485-495, 1992.

10. Smith CC, Vedder LC, Nelson AR, Bredemann TM and McMahon LL: Duration of estrogen deprivation, not chronological age, prevents estrogen's ability to enhance hippocampal synaptic physiology. Proc Natl Acad Sci USA 107: 19543-19548, 2010.

11. Ansari MA and Scheff SW: Oxidative stress in the progression of Alzheimer disease in the frontal cortex. J Neuropathol Exp Neurol 69: 155-167, 2010.

12. Keller JN, Schmitt FA, Scheff SW, Ding Q, Chen Q, Butterfield DA and Markesbery WR: Evidence of increased oxidative damage in subjects with mild cognitive impairment. Neurology 64: 1152-1156, 2005.

13. Zhang W, Wang T, Pei Z, Miller DS, Wu X, Block ML, Wilson B, Zhang W, Zhou Y, Hong JS and Zhang J: Aggregated alpha-synuclein activates microglia: a process leading to disease progression in Parkinson's disease. FASEB J 19: 533-542, 2005. 
14. Gutowicz M: The influence of reactive oxygen species on the central nervous system. Postepy Hig Med Dosw 65: 104-113, 2011 (In Polish).

15. Hu D, Serrano F, Oury TD and Klann E: Aging-dependent alterations in synaptic plasticity and memory in mice that overexpress extracellular superoxide dismutase. J Neurosci 26: 3933-3941, 2006.

16. Dröge W: Free radicals in the physiological control of cell function. Physiol Rev 82: 47-95, 2002.

17. Bruce-Keller AJ, Gupta S, Knight AG, Beckett TL, McMullen JM, Davis PR, Murphy MP, Van Eldik LJ, St Clair D and Keller JN: Cognitive impairment in humanized APPxPS1 mice is linked to $A \beta(1-42)$ and NOX activation. Neurobiol Dis 44: 317-326, 2010.

18. Ansari MA and Scheff SW: NADPH-oxidase activation and cognition in Alzheimer disease progression. Free Radic Biol Med 51: 171-178, 2011.

19. Sorce S and Krause KH: NOX enzymes in the central nervous system: from signaling to disease. Antioxid Redox Signal 11: 2481-2504, 2009.

20. Tejada-Simon MV, Serrano F, Villasana LE, Kanterewicz BI, Wu GY, Quinn MT and Klann E: Synaptic localization of a functional NADPH oxidase in the mouse hippocampus. Mol Cell Neurosci 29: 97-106, 2005.

21. Fan G, Feng C, Li Y, Wang C, Yan J, Li W, Feng J, Shi X and Bi Y: Selection of nutrients for prevention or amelioration of lead-induced learning and memory impairment in rats. Ann Occup Hyg 53: 341-351, 2009.

22. Li WZ, Li WP, Huang DK, Kan HW, Wang X, Wu WY, Yin YY and Yao YY: Dexamethasone and $A \beta_{25-35}$ accelerate learning and memory impairments due to elevate amyloid precursor protein expression and neuronal apoptosis in 12-month male rats. Behav Brain Res 227: 142-149, 2012.

23. Cao Z, Swift TA, West CA, Rosano TG and Rej R: Immunoassay of estradiol: unanticipated suppression by unconjugated estriol. Clin Chem 50: 160-165, 2004.

24. Joffe H, Petrillo LF, Koukopoulos A, Viguera AC, Hirschberg A, Nonacs R, Somley B, Pasciullo E, White DP Hall JE and Cohen LS: Increased estradiol and improved sleep, but not hot flashes, predict enhanced mood during the menopausal transition. J Clin Endocrinol Metab 96: E1044-E1054, 2011.

25. Girouard H, Park L, Anrather J, Zhou P and Iadecola C: Cerebrovascular nitrosative stress mediates neurovascular and endothelial dysfunction induced by angiotensin II. Arterioscler Thromb Vasc Biol 27: 303-309, 2007.

26. Dikalov S, Griendling KK and Harrison DG: Measurement of reactive oxygen species in cardiovascular studies. Hypertension 49: 717-727, 2007.

27. Girouard H, Wang G, Gallo EF, Anrather J, Zhou P, Pickel VM and Iadecola C: NMDA receptor activation increases free radical production through nitric oxide and NOX2. J Neurosci 29 2545-2552, 2009.

28. Hedden T and Gabrieli JD: Insights into the ageing mind: a view from cognitive neuroscience. Nat Rev Neurosci 5: 87-96, 2004.

29. Wilson IA, Gallagher M, Eichenbaum $\mathrm{H}$ and Tanila $\mathrm{H}$ : Neurocognitive aging: prior memories hinder new hippocampal encoding. Trends Neurosci 29: 662-670, 2006.

30. Kondratova AA and Kondratov RV: The circadian clock and pathology of the ageing brain. Nat Rev Neurosci 13: $325-335,2012$

31. Daniel JM, Hulst JL and Berbling JL: Estradiol replacement enhances working memory in middle-aged rats when initiated immediately after ovariectomy but not after a long-term period of ovarian hormone deprivation. Endocrinology 147: 607-614, 2006.

32. Duff SJ and Hampson E: A beneficial effect of estrogen on working memory in postmenopausal women taking hormone replacement therapy. Horm Behav 38: 262-276, 2000.
33. Gibbs RB: Long-term treatment with estrogen and progesterone enhances acquisition of a spatial memory task by ovariectomized aged rats. Neurobiol Aging 21: 107-116, 2000

34. Harman D: Aging: a theory based on free radical and radiation chemistry. J Gerontol 11: 298-300, 1956.

35. Dennis KE, Aschner JL, Milatovic D, Schmidt JW, Aschner M, Kaplowitz MR, Zhang Y and Fike CD: NADPH oxidases and reactive oxygen species at different stages of chronic hypoxia-induced pulmonary hypertension in newborn piglets. Am J Physiol Lung Cell Mol Physiol 297: L596-L607, 2009.

36. Loukogeorgakis SP, van den Berg MJ, Sofat R, Nitsch D, Charakida M, Haiyee B, de Groot E, MacAllister RJ, Kuijpers TW and Deanfield JE: Role of NADPH oxidase in endothelial ischemia/reperfusion injury in humans. Circulation 121: 2310-2316, 2010.

37. Perianayagam MC, Liangos O, Kolyada AY, Wald R, MacKinnon RW, Li L, Rao M, Balakrishnan VS, Bonventre JV, Pereira BJ and Jaber BL: NADPH oxidase p22phox and catalase gene variants are associated with biomarkers of oxidative stress and adverse outcomes in acute renal failure. J Am Soc Nephrol 18: 255-263, 2007.

38. Park KW, Baik HH and Jin BK: IL-13-induced oxidative stress via microglial NADPH oxidase contributes to death of hippocampal neurons in vivo. J Immunol 183: 4666-4674, 2009.

39. Zhang QG, Raz L, Wang R, Han D, De Sevilla L, Yang F, Vadlamudi RK and Brann DW: Estrogen attenuates ischemic oxidative damage via an estrogen receptor alpha-mediated inhibition of NADPH oxidase activation. J Neurosci 29: 13823-13836, 2009.

40. Bruce-Keller AJ, Gupta S, Parrino TE, Knight AG, Ebenezer PJ, Weidner AM, LeVine H, Keller JN and Markesbery WR: NOX activity is increased in mild cognitive impairment. Antioxid Redox Signal 12: 1371-1382, 2010

41. de la Monte SM and Wands JR: Molecular indices of oxidative stress and mitochondrial dysfunction occur early and often progress with severity of Alzheimer's disease. J Alzheimers Dis 9: 167-181, 2006.

42. Inaba S, Iwai M, Furuno M, Tomono Y, Kanno H, Senba I, Okayama H, Mogi M, Higaki J and Horiuchi M: Continuous activation of renin-angiotensin system impairs cognitive function in renin/angiotensinogen transgenic mice. Hypertension 53: 356-362, 2009.

43. Bedard K and Krause KH: The NOX family of ROS-generating NADPH oxidases: physiology and pathophysiology. Physiol Rev 87: 245-313, 2007.

44. Masamune A, Watanabe T, Kikuta K, Satoh K and Shimosegawa T: NADPH oxidase plays a crucial role in the activation of pancreatic stellate cells. Am J Physiol Gastrointest Liver Physiol 294: G99-G108, 2008.

45. Weintraub NL: Nox response to injury. Arterioscler Thromb Vasc Biol 22: 4-5, 2002

46. Raad H, Paclet MH, Boussetta T, Kroviarski Y, Morel F, Quinn MT, Gougerot-Pocidalo MA, Dang PM and El-Benna J: Regulation of the phagocyte NADPH oxidase activity: phosphorylation of gp91phox/NOX2 by protein kinase $\mathrm{C}$ enhances its diaphorase activity and binding to Rac2, p67phox and p47phox. FASEB J 23: 1011-1022, 2009.

47. Mizrahi A, Berdichevsky Y, Casey PJ and Pick E: A prenylated p47phox-p67phox-Racl chimera is a Quintessential NADPH oxidase activator: membrane association and functional capacity. J Biol Chem 285: 25485-25499, 2010.

48. Edlund J, Fasching A, Liss P, Hansell P and Palm F: The roles of NADPH-oxidase and nNOS for the increased oxidative stress and the oxygen consumption in the diabetic kidney. Diabetes Metab Res Rev 26: 349-356, 2010. 